\title{
HARVARD PARTICIPATION IN THE UA1 EXPERIMENT
}

\author{
James W. Rohlf \\ Associate Professor of Physics
}

DOE/ER/40171--1

DE92 013496

\author{
High Energy Physics Laboratory \\ Harvard University \\ Cambridge, MA 02138
}

\begin{abstract}
This is a junior investigator renewal proposal to continue Harvard participation in the UA1 experiment on proton-antiproton collisions.
\end{abstract}

\section{DISCLAIMER}

This report was prepared as an account of work sponsored by an agency of the United States Government. Neither the United States Government nor any agency therecf, nor any of their employees, makes any warranty, express or implied, or assumes any legal liability or responsibility for the accuracy, completeness, or usefulness of any information ${ }_{v}$ apparatus, product, or process disilosed, or represents that its use would not infringe privately owned rights. Reference herein to any specific commercial product, process, or service by trade name, trademark, manufacturer, or otherwise does not necessarily constitute or imply its endorsement, recommendation, or favoring by the United States Government or any agency thereof. The views and opinions of authors expressed herein do not necessarily state or reflect those of the United States Government or any agency thereof. 


\section{Introduction}

The UA1 detector is a multi-purpose $4 \pi$-solid-ıngle device designed for studying proton-antiproton interactions in the Super-Proton-Syncrotron (SPS) Collider at CERN. The UA1 collaboration consists of physicists from Aachen, Annecy, Birmingham, CERN, Harvard, Helsinki, Kiel, Queen Mary College, NIKHEF, Paris, Riverside, Rome, Rutherford, Saclay, Vienna, and Wisconsin. The Harvard physicists participating in UA1 currently include Professor Rubbia (UA1 Spokesman), Dr. Geer, Professor Goodman, Professor Rohlf, and three graduate students Mr. Kroll, Mr. Kwok, and Mr. Schwartz.

\section{Analysis Facilities at Harvard}

In the High Energy Physics Laboratory at Harvard University, we operate a Megatek graphics facility which is connected to our Vax 11/780 computer. The Megatek device has its own memory and microprocessor and enables detailed display and manipulation (through rotations and zooming) of raw and reconstructed data. This graphics tool, which also exists at CERN and other collaborating institutions, has been a crucial element to our understanding of the UAI detector and the physics of $p \bar{p}$ collisions. We also operate a link (via telenet) between the Harvard Vax and the CERN computers giving us ready access (from Harvard) to the CERN central IBM facility, the CERN UA1 Vax, and our Nord computers at the experimental site. This is an inexpensive but slow link which is limited to 1200 baud transmission. This means UA1 events cannot be transferred on this link (a single event would take about an hour); however, one may transfer code and small data sets efficiently, and it serves as a vital communication channel with our CERN collaborators. The link also enables us to edit code and submit jobs on the CERN IBM facility very conveniently.

Activities : July, 11982 - June 30,1983

The first major physics run of the CERN $p \bar{p}$ collider was in 1982. The center-ofmass energy was $540 \mathrm{GeV}$, and an integrated luminosity of $15 \mathrm{nb}^{-1}$ (after dead time) was achieved. The major dicovery from these data was the experimental observation of the charged intermediate vector bosons $\left(\mathrm{W}^{+}\right.$and $\left.\mathrm{W}^{-}\right)$decaying into electron and neutrino. The UA1 experiment found six events with entirely negilible backgrounds. Hadronic jets, as a direct consequence of parton-parton hard scattering, were produced copiously is these data. Di-jet events of invariant mass in excess of $200 \mathrm{GeV} / \mathrm{c}^{2}$ were observed. Detailed measurements of the parton fragmentation into charged particles were made,and the proton (and antiproton) structure functions were also measured at far higher momentum transfer than achieved previously.

Activities : July, 11983 -June 30, 1984

The next data run was in 1983, yielding an integrated luminosity of $136 \mathrm{nb}^{-1}$. The first $Z^{0}$ events were seen in these data decaying into both $\mathrm{e}^{+} \mathrm{e}^{-}$and $\mu^{+} \mu^{-}$. A higher statistics sample of $\mathrm{W}$ events was obtained, enabling a measurement of its mass, spin, and 
asymmetric decay due to parity violating effects of the weak interaction. The decav $W \rightarrow$ $\mu \nu$ was also observed in these data.

The first hint of possible non-standard physics was the observation of events containing $\mathrm{e}^{+} \mathrm{e}^{-} \gamma$ or $\mu^{+} \mu^{-} \gamma$ of invariant mass consistent wich the $Z^{0}$ mass. The probability that these events were due to quantum-electrodynamic (QED) effects was calculated to be very small. A search for $W$-like events containing a massive evy final state produced a null result.

A very exciting area of analysis was an inclusive search for events containing large missing energy. A major part of this analysis was performed at Harvard University, and has procuced some interesting results. We have observed events which contain a single unbalanced hadronic jet which cannot be easily explained through expected processes involving the known quarks and leptons. Although small in number, these events are extremely clean in their signature. Their origin is completely unexplained.

Another class of interesting events was observed containing two muons of the same electric charge.

Activities : July, 11984 - June 30, 1985

This period is the first year of existence of this contract, supporting the operations of Professor Rohlf.

During the period from September to December 1984, the CERN $p \bar{p}$ collider was operated at a total energy of $630 \mathrm{GeV}$. The UA1 experiment recorded $280 \mathrm{nb}^{-1}$ of data on tape. We are currently actively analyzing these data at Harvard. We have detected about 200 leptonic $W$ decays and 20 leptonic $Z^{0}$ decays. We have also seen clear evidence for the decay $W \rightarrow \tau \nu$ in which the $\tau$-lepton appears in the UA1 detector as a hadronic jet with charged multiplicity of 1 or $3 \mathrm{cf}$ low invariant mass. We have now observed the W decay into all known leptons $(e, \mu$, and $\tau$ ). The most exciting and active area of research is ana!ysis of events with large missing transverse energy. This is actively going on now at Harvard.

We have put together a Fastbus system of TDC's for readout of our new micro-vertex detector. The major inovative component is a special Fastbus-VME interface designed and built at Harvard. This enables readout of the Fastbus memory at $10 \mathrm{MHz}$. (The Fastbus components needed for this project were purchased through our collaborators at Riverside and the VME components are on loan from CERN.)

We have also been particularly active in the design of a depleted uranium calorimeter for UA1. The Harvard group has had responsibility for the specification and the procurement of 300 tons of depleted uranium for this project.

During March the collider will have a data run in a pulsed mode $2 t 900 \mathrm{GeV}$ total energy. Due to the duty cycle of the magnet ramping and the limitation of the focusing 
quadrupoles in the SPS, only low luminosities are expected. UA1 (together with UA5) will take data during this run.

Proposed Activities : July 1, 1985 -June 30, 1986

During the first part of this period we expect to actively continue analysis on the present data. Particular emphasis will be placed on events with large missing energy and $\mathrm{W}$ and $\mathrm{Z}^{0}$ decays.

The next high luminosity run $(630 \mathrm{GeV})$ of the $p \bar{p}$ collider at CERN will start in August 1985. Based on the excellent performance of the accelerator during the 1984 run, we may expect an integrated luminosity of more than three times the previous data. This would yield about 600 decays $\mathrm{W} \rightarrow \mathrm{e} \nu$ and 60 decays $\mathrm{Z}^{0} \rightarrow \mathrm{e}^{+} \mathrm{e}^{-}$and a similar number of muonic decays. We will have correspondingly higher sensitivity to detection of possible rare decay modes of intermediate vector bosons.

The Cern computer center will not be able to handle the processing of the data expected in 1985. Indeed, our ability to extract physics results from the data currently in hand, is now limited by the amount of computing time available. We intend to upgrade the Harvard High Energy Physics Laboratory computing facility with a new host computer and dedicated 3081 emulators. Such a facility would enable us to process a significant fraction of UA1 raw data at Harvard, a capability which exists currently within the UA1 collaboration only at CERN. A link is forseen to our collaborators at Riverside and Wisconsin. These emulators are currently being built at CERN (CERN/SLAC collaboration) and will also be installed as part of the online triggering system in UA1. Equipment money is requested in this proposal to build a 3081 emulator for use in data analysis at Harvard.

A major upgrade of the CERN $p \bar{p}$ facility has been approved and is under construction. The key component of this machine inprovement program is the construction of an antiproton collector ring (ACOL). The projected luminosity gain from ACOL is about a factor of 40. This will give instantaneous luminosities of nearly $10^{31} \mathrm{~cm}^{-1} \mathrm{sec}^{-1}$. We then anticipate being able to observe tens-of-thousands of intermediate vector boson decays with a much improved detector. Of course, as the luminosity increases, we have greater sensitivity to higher mass quark-antiquark collisions. We then effectively have a higher energy machine when viewed at the proton constituent level.

The first part of the UA1 hardware upgrade includes the addition of new muon chambers and a vertex detector. Most of the new muon chambers are now in place and the vertex detector is scheduled to be installed in Summer 1985. The second part of the UA1 upgrade is the replacement of the lead portion of our calorimeter with uranium. The uranium device gives an equal response for electromagnetic and hadronic showers, which leads to superior energy resolution for jets. The new calorimeter will also have superior spacial resolution. It is scheduled for installation in 1986. 

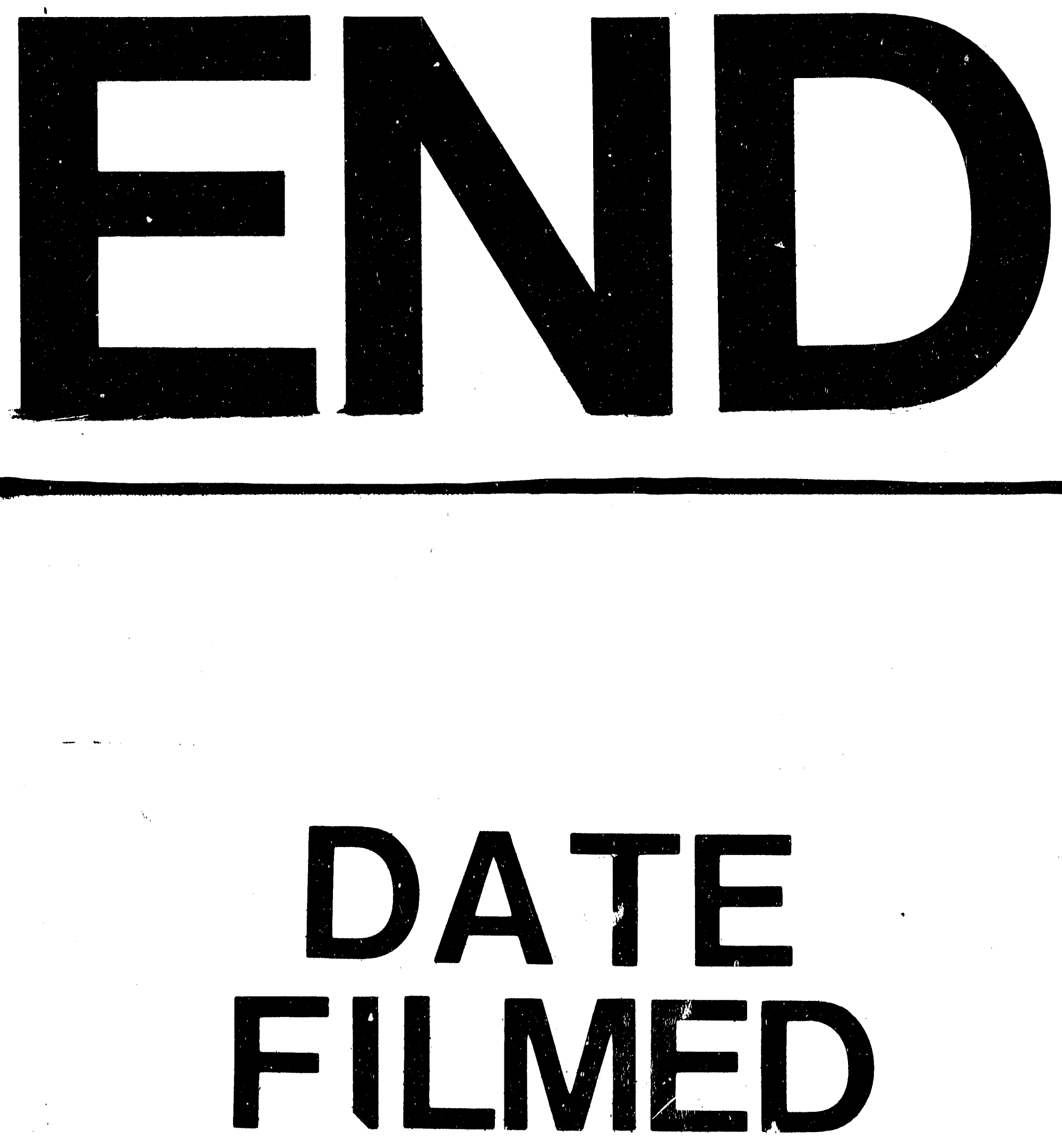

$6 / 23 / 92$ 
프

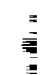

$\underline{\underline{\underline{\underline{z}}}}$ 雎 